\title{
Implementation Of Patient-Centered Care In Adult Inpatient Unit At Dr. H. Moch. Ansari Saleh Hospital Banjarmasin
}

\author{
Yunina Elasari ${ }^{1}$, Elly Nurachmah ${ }^{2}$, Yustan Azidin ${ }^{3}$ \\ \{ayiedeh@gmail.com ${ }^{1,2,3}$ \} \\ Universitas Sari Mulia Banjarmasin ${ }^{1,2}$ \\ Universitas Muhammadiyah Banjarmasin ${ }^{3}$
}

\begin{abstract}
The implementation of patient-centered care is one of the goals to improve health services to be more effective and efficient because good quality health services can improve health progress in all counrties. This study aimed to show implementation of patient-centered care. The research method used a descriptive quantitative involving 100 patients in adult inpatient units who were selected with proportionally stratified random sampling. The data were analyzed using normality distribution tests. The results showed the average implementation value of the patient-centered care 88,77 with the highest value 131. The results suggested that the nursing management can make efforts to optimize the implementation of the patient-centered care dimension in the hospital.
\end{abstract}

Keywords: patient-centered care, patient preference, patient involvement

\section{Introduction}

In current condition, health services are no longer focused on disease but are patientcentered or known as patient-centered care (PCC). PCC is implemented in order to improve the system and quality of health services [9]. Institute of Medicine defines PCC as providing care that is respectful of and responsive to, individual patient preferences, needs and values, and ensuring that patient values guide all clinical decisions. Patient-centered care is broadly described as an approach to care that puts individuals at the center of care [13]. PCC known as the most important dimension of high quality health care [7].

Research conducted by the Picker Institute and Harvard Medical School identified eight dimensions of Patient-Centered Care known as the Eight Picker Principles of Patient Centered Care namely patient preferences, coordination of care, information and education, physical comfort, emotional support, family and friends, continuity and transition, and access to care $[2]$.

Dimension of patient preferences by maintaining dignity and showing sensitivity to cultural values, providing information and involving them in making decisions [2]. Patient 
involvement in expressing preferences will prevent unmet needs and also have an impact on reducing quality of life [10].

Dimension of coordination of care is that various health service providers coordinate and integrate care and clinical services to reduce negative feelings in patients [2]. The findings indicate the importance of care coordination to prevent major impacts from complex health problems and interventions focused on providing holistic based care [17].

Dimension of information and education is to provide complete information to patients about the clinical status, progress and prognosis of the disease, treatment process, and information to help independence and to promote their health [2].

Dimension of physical comfort is provided by increasing the comfort of the patient during treatment, especially those related to pain management and the hospital environment (eg privacy, cleanliness, comfort, and accessibility to visits) [2].

Dimensions of emotional support that is by helping to relieve fear and anxiety from physical conditions, treatment, and prognosis; the impact of their illness on themselves, their families, and those closest to them; and the financial impact of their illness [2]. The results showed that the empathy approach in PCC can reduce preoperative anxiety and improve surgical recovery, surgical wound healing and patient satisfaction [12].

Dimension of family and friends involvement is by accommodating family and friends in providing support during patient care, respecting the role of family and friends in making decisions, and supporting family members in recognizing patient needs [2].

Dimensions of continuity and transition that is reducing patient anxiety about their ability to manage themselves to their health after discharge [2].

Dimension of access to care is ensuring access to various health services, availability of transportation, ease of scheduling to meet health workers, access to specialist or special services when needed [2]. Health workers are important to understand the obstacles experienced by individuals when accessing health care and this is as important as documenting the type of care that is delayed due to obstacles [18].

The concept of PCC is implemented by hospitals in Indonesia through the 2012 KARS version of hospital accreditation standards. Hospital accreditation is a major factor for implementing PCC as a strategy for continuous quality improvement [8]. PCC is a modern, innovative approach and has become a global trend in hospital services [15]. The implementation of PCC to all health workers is an important part of providing daily services. The literature review shows that nurses play the most important role in the implementation of PCC because nurses are located 24 hours by the patient's bedside [14]

The results of interviews with nurses said that the concept of PCC was implemented in providing nursing services to patients but was not optimal. Health care providers must ensure that patients are given the information needed to make decisions and are supported in every decision making process. The results showed that the implementation of PCC was beneficial for patients which influenced lifestyle changes more positively. Thus, when given support, 
education and information, patients can apply this information to better manage their health and evaluate their own health status [4]

\section{Methods}

The design of this study uses a quantitative description with a cross sectional approach. The number of samples involved 100 patients in the adult inpatient at H. Moch. Ansari Saleh Banjarmasin Hospital selected by proportionate stratified random sampling. The implementation of patient-centered care in adult inpatient care was measured using the Patient-Centered Care Improvement Guide instrument [6] consisting of 34 statement items. This instrument has been tested the instrument has a validity value $\geq 0,361(\mathrm{n}=30)$ and a reliability value of 0,931 . Univariate analysis of patient-centered care is done using central (numerical) tendencies.

\section{Results}

An illustration of the implementation of the patient-centered care dimension. The implementation of patient-centered care dimensions shows that some patients in the study felt the implementation of patient-centered care conducted by nurses with an average value of 88,77 (highest value 131) which includes dimensions of patient preferences with an average value of 6,90 (highest value 12); dimensions of coordination of care with an average value of 9,71 (highest value 16); dimension of information and education with an average value of 12,33 (highest value of 20); physical comfort dimensions with an average value of 9,76 (highest value 12); dimensions of emotional support with an average value of 13,51 (highest score of 20); dimensions of family and friend involvement with an average value of 13,51 (highest score of 20); dimensions of , continuity and transition with an average value of 11,52 (highest value of 20); and dimensions of access to care with an average value of 10,75 (highest value of 16) (see table 1).

Table 1. Distribution of the implementation of the patient-centered care dimension $(\mathrm{N}=100)$

\begin{tabular}{lccccc}
\hline \multicolumn{1}{c}{ Variabel } & Mean & Median & SD & Min-Mak & 95\% CI \\
\hline Patient-Centered Care & 88,77 & 89 & 19,505 & $41-131$ & $84,90-92,64$ \\
Patient preferences & 6,90 & 7 & 2,762 & $3-12$ & $6,35-7,45$ \\
Coordination of care & 9,71 & 9,50 & 3,092 & $5-16$ & $9,10-10,32$ \\
Information and education & 12,33 & 12,50 & 3,822 & $5-20$ & $11,57-13,09$ \\
Physical comfort & 9,76 & 10 & 1,876 & $5-12$ & $9,39-10,13$ \\
Emotional support & 13,51 & 14 & 3,32 & $6-20$ & $12,85-14,17$ \\
Family and friends & 13,51 & 14 & 4,28 & $5-20$ & $12,66-14,36$ \\
involvement & 11,52 & 13 & 5,555 & $5-20$ & $10,42-12,62$ \\
Continuity and transition & 10,75 & 11 & 3,083 & $4-16$ & $10,14-11,36$ \\
Access to care & & & & & \\
\hline
\end{tabular}

\section{Discussion}

Implementation of patient-centered care dimensions in the inpatient hospital Dr. H. Moch. Ansari Saleh Banjarmasin obtained a portion of patients who have undergone patient- 
centered care performed by nurses. The implementation of the dimension patient preferences by patients in adult inpatient settings that is the patient feels the information provided by the nurse about their preferences and treatment priorities, patient needs, and assist patients in making decisions regarding their care.

Some nurses involve patients in nursing planning as well as in making decisions of nursing actions to be performed to the patients. Patients and families are involved in determining treatment priorities and treatment plans according to their preferences, not from the service providers, and reformulate plans when the situation has changed [5]. The importance of implementing this dimension according to the results show that the involvement of patients in conveyed the preferences will prevent unmet needs and also have an impact on decreasing quality of life [10].

Implementation of the coordination of care dimension, namely patients feel the coordination and integration of care and clinical services from nurses to reduce fear in patients. In the opinion of researchers, some nurses have coordinated and integrated services with other health workers well in an effort to improve patient health. Care provided by nurses, doctors, and other health workers must work together in implementing patient-centered care to meet the needs of patients with complex problems in various social situations / various chronic social situations [17]. Chronic, an increase in the number of health care providers, a large number of treatment sites, and duration of care become shorter so that the transition of all care becomes very important [5]. Integrating and coordinating patient care activities aim to produce efficient care processes, more effective use of human and other resources, and the possibility of better patient care outcomes.

Implementation of the dimensions of information and education, the patient feels the nurse provides complete information about the clinical situation, progress, prognosis, and treatment process; and to make the patient independent. Some nurses have provided information regarding the process and results of care to patients in full. Some patients do not get the health education they need. This can occur because some nurses have less knowledge related to patient health problems so they cannot provide information that is appropriate to the needs of patients. The implementation of the dimensions of information, communication, and education in the implementation of patient-centered care according to researchers is closely related to the results of good quality care.

Implementation of the dimensions of physical comfort, the patient feels physical comfort during treatment related to pain management and comfort. In the opinion of researchers, some nurses always provide physical comfort such as helping to reduce pain and discomfort in patients. The results show that pain management in patients before surgery can increase patient knowledge to prevent negative outcomes such as chronic pain that have an impact on increasing the duration of hospitalization of patients [11].

Implementation of the dimensions of emotional support, the patient felt a decrease in fear and anxiety over his illness because nurses provide support to them. In the opinion of researchers, some nurses are always sincere in listening to patient complaints and provide motivation in every process of care. Patients and families may experience anxiety and difficulties caused by various problems, so the underlying factors need to be identified and addressed. Nurses need to make different contributions to each patient [5]. Patients and 
families may experience anxiety and difficulties caused by various problems, so the underlying factors need to be identified and handled. Nurses need to make different contributions to each patient [5]. Empathy and patient-centered care has been linked to better patient outcomes. Empathy and patient-centered care has been linked to better patient outcomes. Empathy is also associated with positive outcomes such as pain relieve and anxiety reduce and a number of studies have examined the correlation between empathy and quality of life [19].

Implementation of the dimensions of the family and friends involvement, the patient felt that the nurse accommodating family and friends in providing support during treatment, advocating for decision making, and recognizing the patient's needs. In the opinion of researchers, some nurses always involve the patient's family and friends in accompanying and helping to meet the needs of patients while in hospital and involve family and friends of patients in making decisions related to patient care. Family involvement in care and decision making improves the experience of patients and families in the rehabilitation process [9].

Implementation of dimensions of Continuity and transition, the patient felt that the nurse provides information about how the patient manages his health after returning home, about drugs, physical limitations, nutrition and access to ongoing services to patients. In the opinion of researchers, some nurses have provided information related to treatment plans after patients go home and conveyed things that should be avoided after patients go home, but some patients said nurses had not provided any information regarding care after returning home.

In the opinion of researchers, the factors that influence the implementation of this dimension are because not all nurses are responsive to patient problems and do not involve full the patient participation during treatment. In addition, the lack of knowledge of some nurses related to patient health problems is a barrier to the implementation of patient-centered care in this dimension. Health education must start from the start of care to empower patient participation in planning and decision making about their care. Planning the patient discharge process can take a while, so the assessment and planning process can begin as soon as the patient is admitted as an inpatient [9]. The results of the study showed that ongoing care for patients with Chronic Obstructive Pulmonary Disease (COPD) can reduce repeated visits of patients after discharge from the hospital [16].

Implementation of dimensions of access to care, namely patients feel certainty from nurses regarding access to various health services, availability of transportation, ease of scheduling to meet health workers, and access to special services if needed.

In the opinion of researchers, some nurses bridge the ease of access to health insurance services and inform access to service facilities, but some patients say nurses never inform the doctor's visit time and tell patients to call the nurse or ring the bell if needed help. According to researchers, factors that influence the implementation of the access dimension to services are the less of adequate facilities related to access to services and the lack of nurse communication in facilitating such access.

The results of the study showed that patients who do not have access to health services have a lower chance of knowing their prediabetic status compared to those who have full access and patients with insurance but who do not have routine care have the greatest chance 
of not knowing prediabetes [3]. This shows the importance of nurses to provide ease of access to services in order to prevent patients from adverse health effects.

\section{Conclusions}

Some patients in the study felt the implementation of patient-centered care conducted by nurses which included dimensions of respecting the values, choices, and needs conveyed by patients; dimensions of coordination and integration of care; dimension of information, communication and education; dimensions of physical comfort; dimensions of emotional support and decreased fear and anxiety; dimensions of family and friend involvement; dimensions of ongoing care and a smooth transition; and dimensions of access to services. Implementation of the patient-centered care dimension is important in improving the quality of health services with better patient health outcomes so that hospital management can make efforts to optimize the implementation of the patient-centered care dimension in the clinic.

\section{References}

[1] Bamm E.L., Peter R., Seanne W., Paul S., \& Nadilein, M: Exploring Client-Centered Care Experiences in In-Patient Rehabilitation Settings. Global Qualitative Nursing Research. (2015)

[2] Baning, K.M.: Outcomes of a Comprehensive Patient and Family-Centered Care Program in An Adult Intensive Care Unit. Dissertation, the University of Arizona (2012)

[3] Campbell, T.J., Alberga, A., Rosella, L.C. (2016). The impact of access to health services on prediabetes awareness: A population-based study. Preventif Medicine, 93, pp. 7-13.

[4] Delaney, L: Patient-Centered Care as an Approach to Improving Health Care in Australia Collegian. pp. 119-123. (2018)

[5] Drenkard, K., N. (2013). Patient-Centered Care. American Nurses Credentialing Center.

[6] Frampton, S., Sara, G., Carrie, B., Maria, H., Sheryl, H., Susan, B.S., \& Susan, S. (2008). Patient-Centered Care Improvement Guide. Camden: Planetree, Inc.

[7] Gill, S.D., Dunning, T., McKinnon, F., Cook, D., \& Bourke, J.: Understanding the Experience of Inpatient Rehabilitation: Insights into Patient-Centered Care form Patients and Family Members. pp. 264-272. Scandinavian Journal of Carirng Sciences (2014)

[8] Hijazi, H.H., Heather, L.H., Mohammad, S.A., Hussam, A.A., Rabah, M.A., \& Sanjai, K.P.: The Impact of Applying Quality Management Practices on Patient Centeredness in Jordanian Public Hospitals: Results of Predictive Modeling. pp. 1-15. The Journal of Health Care Organization, Provision, and Financing (2018)

[9] Institute of Medicine: Crossing the Quality Chasm:A New Health System for the $21^{\text {st }}$ Century. National Academy Press, Washington DC (2001)

[10] Johnson, F.R. \& Zhou, M.: Patient Preferences in Regulatory Benefit-Risk Assessments: A US Perspective. Vol. 19, pp. 741-745. Value Health (2016)

[11] O’Donnell. (2018). Preoperative Pain Management Education: An Evidence-Based Practice Project. Journal of Perianesthesia Nursing, 33 (6), pp. 956-963

[12] Pereira, L., Margarida, F., \& Irene, P.C.: Preoperative Anxiety in Ambulatory: The impact of an Empathic Patient-centered Approach on Psychological and Clinical Outcomes. Patient Education and Counseling (2015) 
[13] Sidani, S. \& Marry, F.: Patient-centered care: Clarification of its Specific Elements to Facilitate Interprofessional Care. Vol 2, pp. 134-141. Economic and Social Research Institute (2014)

[14] Small D. \& Small R.: Patients first: engaging the hearts and minds of nurses with a patient-centered practice model. Vol. 2. OJIN: The Online Journal of Issues in Nursing (2011)

[15] Sutoto: Konsep dan Prinsip Pelyanan Berfokus pada Pasien dalam Standar Akreditasi Versi 2012 (2014)

[16] Swanson, J.O., Verena, V., Leonie, S., Terje, P.H., \& Tron, A.M. (2018). Continuity of Care and Its Effect on Readmissions for COPD Patients: Comparative study of Norway and Germany. Health Policy, 122, pp. 737-745.

[17] Vanderboom, C.E., Thackeray, N.L., \& Lori, M.R.: Key Factor in Patient-Centered Care Coordination in Ambulatory Care: Nurse Care Coordinators' Perspectives. pp 18-24. Applied Nursing Research, (2015)

[18] Wong, J.L., Kevin, N.A., Tracy, M.M., Kimberly, P.H., \& Ivan, M.: Identification of Targets for Improving Access to Care in Persons with Long Term Physical Disabilities. Disability and Health Journal (2019)

[18] Ye, Y., Dengyan, Ma., Huaihong, Y., Lin, C., Guorong, W., Jing, S., Yan, Y., Yu, G., \& Xiaolian, J. (2019). Moderating Effects of Forgiveness on Relationship Between Empathy and Health-Related Quality of Life in Hemodialysis Patient: A Structural Equation Modeling Approach. Journal of Pain and Symptom Management, 57, pp, 224 232 